\title{
3D MR Neurography of the Lumbosacral Plexus: Obtaining Optimal Images for Selective Longitudinal Nerve Depiction
}

\author{
(D) G. Cho Sims, (D) E. Boothe, (D) R. Joodi, and (D) A. Chhabra
}

\begin{abstract}
BACKGROUND AND PURPOSE: The number of centers currently performing 3D fat-suppressed isotropic imaging is limited. If the angular orientations of the major lumbosacral plexus nerves on 3D isotropic MR neurography could be determined, similar planes could be prescribed during acquisition of $2 \mathrm{D}$ or $3 \mathrm{D}$ nonisotropic techniques for optimal depiction of various nerves. Our aim was to determine oblique sagittal and coronal angular measurements for longitudinal depiction of lumbosacral plexus nerves. Interobserver and intraobserver performance and mean calibers of sciatic and femoral nerves were also determined.
\end{abstract}

MATERIALS AND METHODS: A consecutive series of lumbosacral plexus MR neurography examinations with 3D nerve-selective imaging performed during a 10-month period on a 3T scanner were evaluated. Two observers performed reconstructions and angular measurements. Sciatic and femoral nerve diameters were measured. Descriptive statistics and intraclass correlation coefficient correlations were used.

RESULTS: There were 52 subjects, 11 men and 41 women. Mean sagittal thecal sac angles for coronal demonstration of lumbosacral plexus nerve roots from $\mathrm{L} 1$ to $\mathrm{S} 1$ for 2 independent observers measured $13.58^{\circ} \pm 2.87^{\circ}$ and $13.61^{\circ} \pm 2.18^{\circ}$. Mean sagittal femoral nerve angles were $27.78^{\circ} \pm 4.81^{\circ}$ and $28.94^{\circ} \pm 4.49^{\circ}$, and mean sagittal sciatic nerve angles were $-10.7^{\circ} \pm 3.75^{\circ}$ and $-11.82^{\circ} \pm 2.87^{\circ}$. Coronal angular measurements of the femoral and sciatic nerves were similar. The intraclass correlation coefficient was moderate (0.582-0.671) for interobserver performance. For intraobserver performance among various angular measurements, the intraclass correlation coefficient was moderate to good $(0.586-0.788)$. Femoral nerve caliber on MR imaging was almost half that of the sciatic nerve. Mean right femoral nerve thickness was $4.52 \pm 1.11 \mathrm{~mm}$ and $4.85 \pm 0.64 \mathrm{~mm}$ for the 2 observers, and mean left femoral nerve thickness was $4.48 \pm 0.97 \mathrm{~mm}$ and $4.94 \pm 0.57 \mathrm{~mm}$. Mean right sciatic nerve thickness was $9.71 \pm 1.76 \mathrm{~mm}$ and $9.94 \pm 0.83 \mathrm{~mm}$, and mean left sciatic nerve thickness was $10.03 \pm 1.71 \mathrm{~mm}$ and $9.98 \pm 0.99 \mathrm{~mm}$.

CONCLUSIONS: Angular lumbosacral plexus measurements aid in the prescription of different planes on MR imaging for the optimal longitudinal demonstration of nerves.

ABBREVIATIONS: ICC = intraclass correlation coefficient; LS = lumbosacral; SHINKEI = nerve-SHeath signal increased with INKed rest-tissue rarE Imaging

$\mathrm{T}$ he lumbosacral (LS) plexus is a complex network of nerves, which provides both motor and sensory innervation to most structures of the pelvis and lower extremities. Most anatomy illustrations in the text books and Internet Web sources depict coronal views of the LS plexus and its branch nerves to demonstrate their longitudinal extent. Some surgeons and physicians prefer to visualize the LS plexus in the coronal

Received April 13, 2016; accepted after revision May 25.

From the Department of Musculoskeletal Radiology, University of Texas Southwestern Medical Center, Dallas, Texas.

Please address correspondence to Avneesh Chhabra, MD, Department of Musculoskeletal Radiology, UT Southwestern Medical Center, 5323 Harry Hines Blvd, Dallas, TX 75390-9178; e-mail: avneesh.chhabra@utsouthwestern.edu

http://dx.doi.org/10.3174/ajnr.A4879 plane, and this plane may be beneficial for patient consultations. MR imaging provides the best soft-tissue contrast for the evaluation of deep soft-tissue structures, including the nerves. ${ }^{1}$ With increasing frequency, MR neurography is playing a prominent role in the diagnosis, characterization, localization, and determination of the extent of pathology in patients with symptoms of lumbar plexopathy. ${ }^{2,3}$ Due to the complex anatomy and oblique course of the plexus branches, however, the longitudinal extent of pathology is difficult to determine on direct coronal or sagittal MR images. 3D isotropic MR images can be reconstructed in various arbitrary planes to depict the peripheral nerves in their entirety. ${ }^{4}$ However, the number of centers currently performing 3D fat-suppressed isotropic imaging is limited due to various hardware and software limitations. If the angular orientations of the major LS plexus nerves 

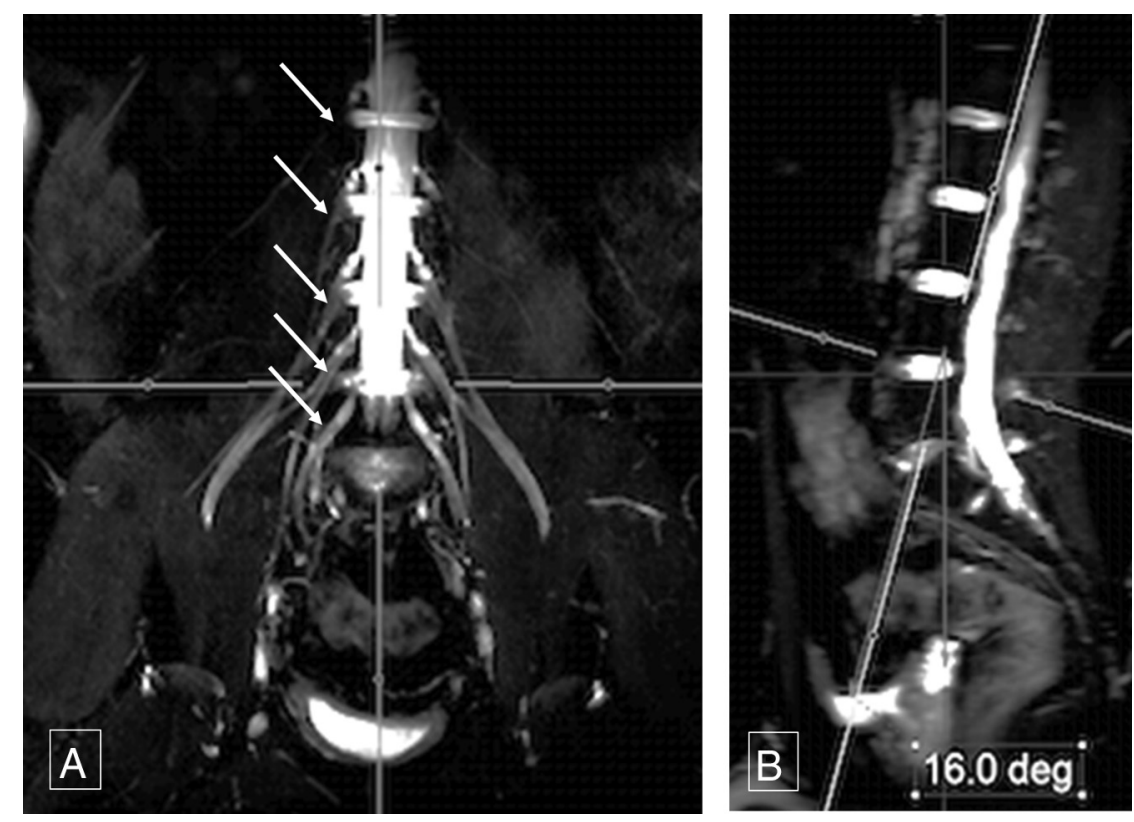

FIG 1. Sagittal thecal sac angle. Thick-slab 20-mm coronal MIP image from a 3D SHINKEl acquisition $(A)$ obtained at a sagittal angle of $16^{\circ}(B)$ optimally demonstrates all the lumbar nerve roots (arrows, $A$ ) from their origin to the maximum length. Deg indicates degree.
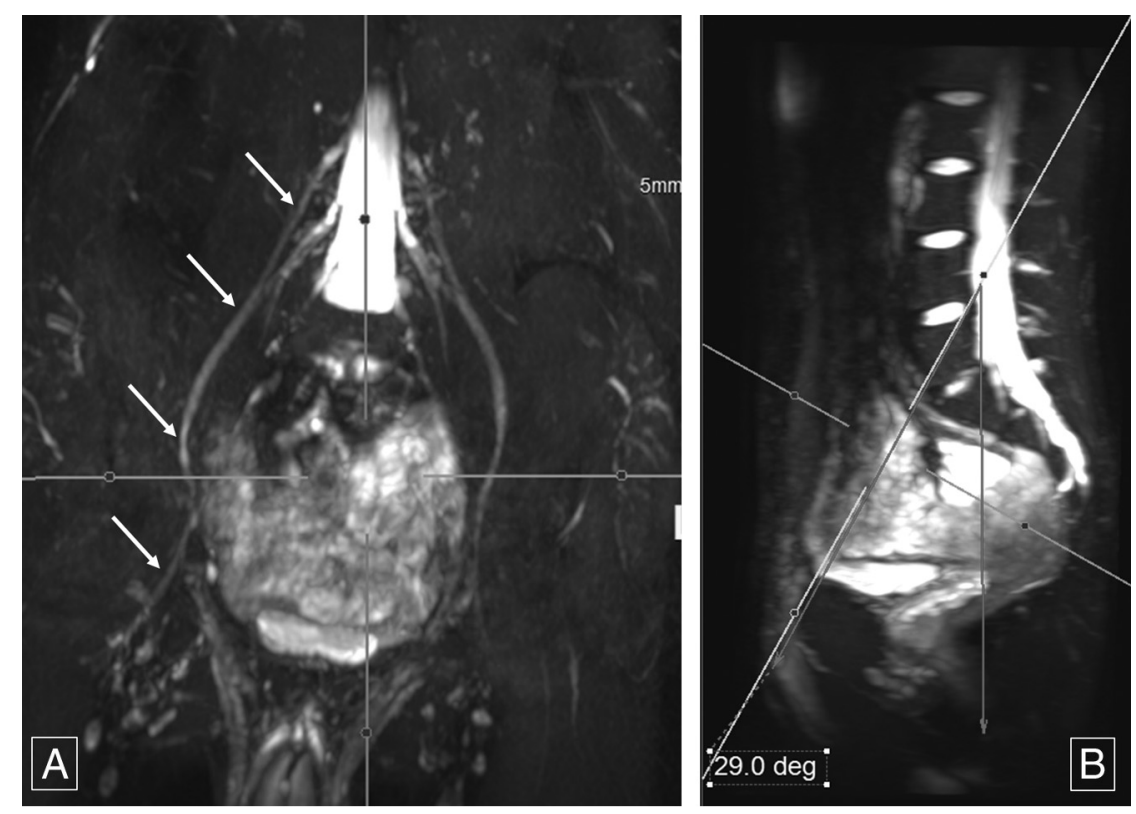

FIG 2. Sagittal femoral nerve angle. Thick-slab 20-mm coronal MIP image from a 3D SHINKEl acquisition (A) obtained at a sagittal angle of $29^{\circ}(B)$ optimally demonstrates bilateral femoral nerves (arrows, A) from their origin to the maximum length across the inguinal ligaments. Deg indicates degree. nerves from their origin to the most distal extent without a break in continuity. As secondary aims, interobserver and intraobserver performance was assessed and the average calibers of sciatic and femoral nerves were measured to generate normative data.

\section{MATERIALS AND METHODS}

Informed consent for this retrospective evaluation was waived, and anonymized data were evaluated. A consecutive series of LS plexus MR neurography examinations for pelvic pain performed during a 10-month period (November 2013 to August 2014) on a 3T scanner (Achieva; Philips Healthcare, Best, the Netherlands) were evaluated. The inclusion criteria were consecutive examinations with no distortion of images by metal, motion, or poor fat suppression. The exclusion criteria were incomplete imaging or evidence of any major nerve pathology, which could cause abnormal thickening in the evaluated nerves. All the MR imaging examinations were performed on nerve-SHeath signal increased with INKed rest-tissue rarE Imaging (SHINKEI; Philips Healthcare) acquisitions, which were obtained as part of the LS plexus MR neurography examination. This sequence uses adiabatic inversion recovery fat suppression for uniform fat saturation and a motionsensitive driven equilibrium pulse for vascular signal suppression for selective demonstration of the LS plexus nerves. ${ }^{5}$ The SENSE XL Torso coil (Philips Healthcare) combined with spine elements was used for imaging. The parameters of the sequence included the following: TR, $2000 \mathrm{~ms}$; TE, $78 \mathrm{~ms}$; voxels, $1.5 \mathrm{~mm}$ isotropic; acquisition time, 7-8 minutes; fat suppression, spectral adiabatic inversion recovery. The source data were manipulated on independent Aquarius iNtuition software (TeraReon 3D isotropic MR neurography could be determined, similar planes could be prescribed during acquisition of $2 \mathrm{D}$ or $3 \mathrm{D}$ nonisotropic techniques for optimal depiction of various nerves, depending on the clinical suspicion of different neuropathies. This added oblique imaging plane/sequence would help the radiologists and referring physicians precisely assess the location and extent of the pathology while facilitating multidisciplinary communication.

The primary aim of the study was to determine oblique sagittal and coronal angular measurements for the optimal longitudinal depiction of lumbosacral plexus nerve roots and sciatic and femoral con, San Mateo, California). Two observers (E.B. and R.J., second- and third-year radiology residents) performed the reconstructions and measurements independently, following training thick-slab maximum-intensity reconstructions were performed, which allowed maximum longitudinal visualization of the LS plexus nerve roots and sciatic and femoral nerves from their origin to termination without a break in continuity (ie, the distalto-inguinal ligament in the case of femoral nerves). Thinner slab MIP reconstructions did not show the maximum longituon an initial set of 10 cases. Sagittal and coronal oblique $20-\mathrm{mm}$ - 

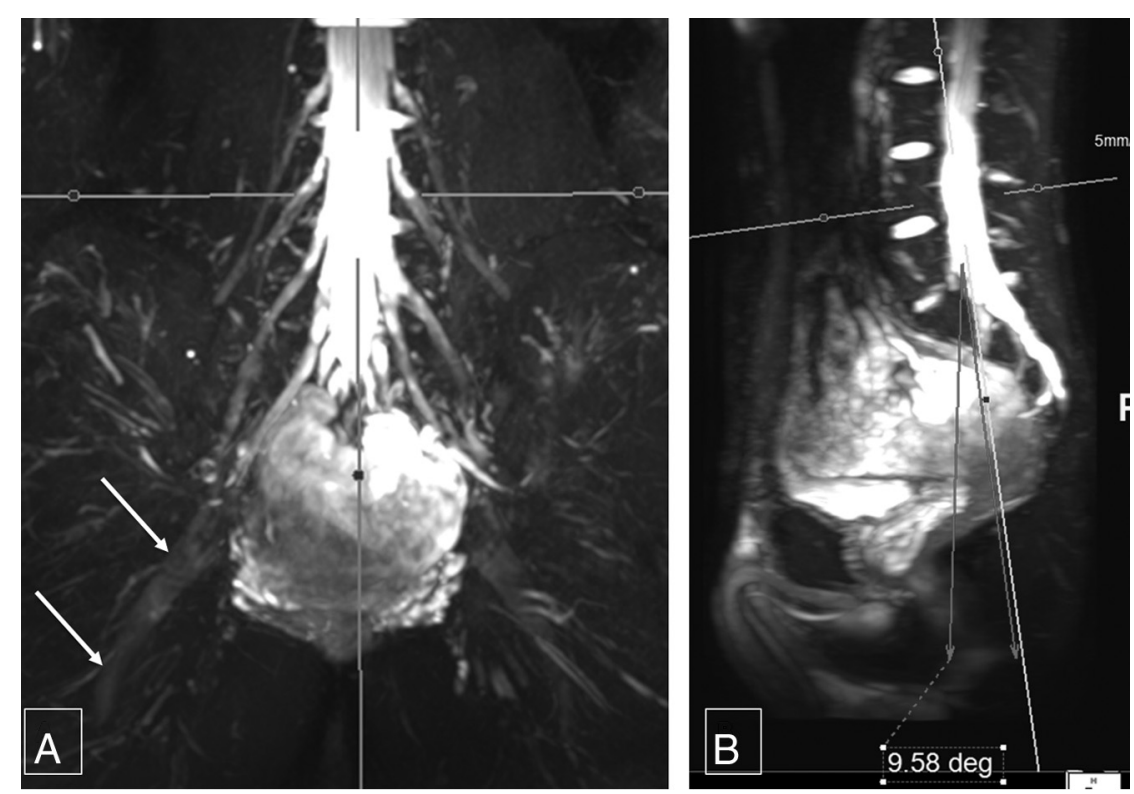

FIG 3. Sagittal sciatic nerve angle. Thick-slab $20-\mathrm{mm}$ coronal MIP image from a 3D SHINKEI acquisition $(A)$ obtained at a sagittal angle of $-9.6^{\circ}(B)$ optimally demonstrates the bilateral sciatic nerves (arrows, $A$ ) from their origin to the maximum length. Deg indicates degree.
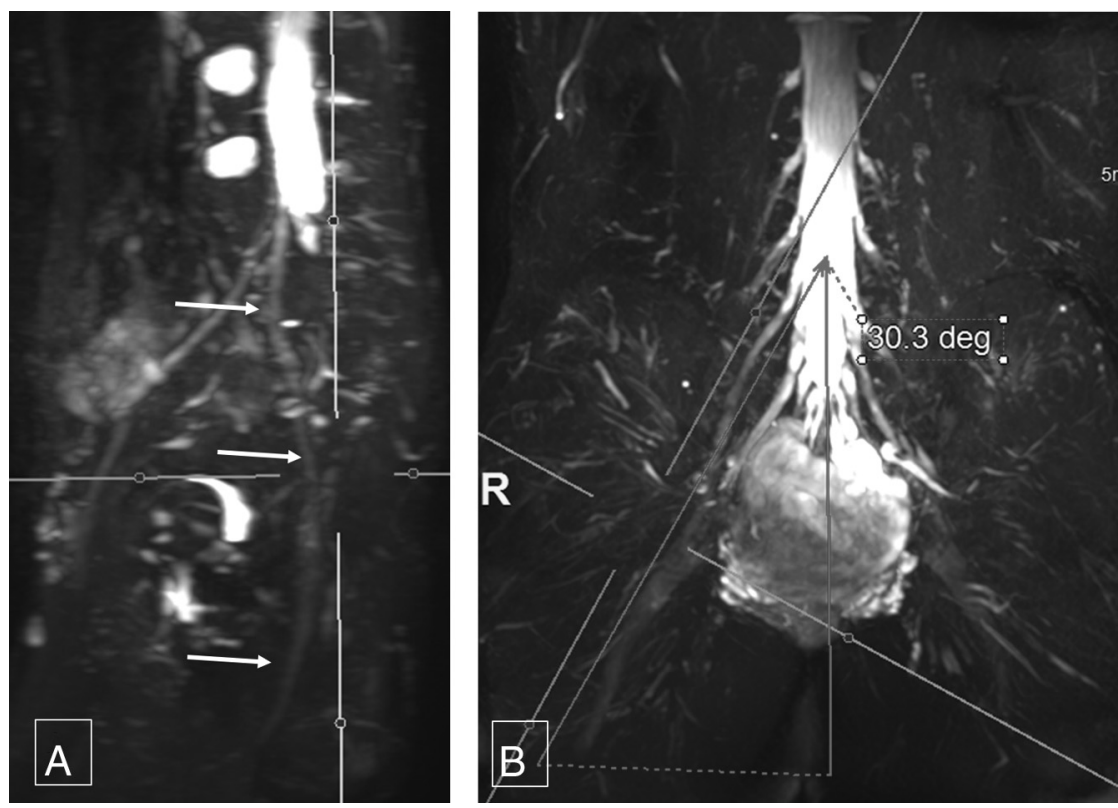

FIG 4. Coronal sciatic nerve angle. Thick-slab $20-\mathrm{mm}$ sagittal MIP image from a 3D SHINKEI acquisition $(A)$ obtained at a coronal angle of $30.3^{\circ}(B)$ optimally demonstrates the right sciatic nerve (arrows, $A$ ) from its origin to the maximum length. Deg indicates degree. pelvis, respectively, at the site of optimal nerve visualization and maximum thickness (ie, immediately below the piriformis muscle and sciatic notch for the sciatic nerve and adjacent to the iliopsoas crotch for the femoral nerve). Descriptive statistics were performed, and data were expressed as mean $\pm \mathrm{SD}$. Interobserver variance was evaluated by using the intraclass correlation coefficient (ICC), with values of $\geq 0.65$ considered good; $<0.65$ to $\geq 0.50$, moderate; $<0.50$ to $\geq 0.40$, fair; and $<0.40$, poor correlations. Intraobserver performance was also assessed by using the ICC for the reader E.B., who obtained repeat measurements approximately 25 weeks following the initial measurements.

\section{RESULTS}

There were 62 examinations with full abdomen and pelvis MR neurography imaging, though 10 were excluded due to hardware artifacts $(n=3)$ and motion and suboptimal fat suppression and incomplete imaging $(n=7)$. Final samples included 52 subjects, 11 men (mean age, $45.73 \pm 18.4$ years) and 41 women (mean age, $48.85 \pm 12.65$ years). All reconstructions were successfully obtained in $<7$ minutes.

Mean sagittal thecal sac angles for the coronal demonstration of LS plexus nerve roots from L1 to S1 for the 2 independent observers measured $13.58^{\circ} \pm 2.87^{\circ}$ and $13.61^{\circ} \pm 2.18^{\circ}$ (Fig $1)$. For complete longitudinal nerve course depiction bilaterally in the coronal plane, the mean sagittal angular measurements of the femoral nerve were $27.78^{\circ} \pm 4.81^{\circ}$ and $28.94^{\circ} \pm 4.49^{\circ}$ (Fig 2). Similarly, mean sagittal sciatic nerve angle measurements for coronal depiction were $-10.7^{\circ} \pm 3.75^{\circ}$ and $-11.82^{\circ} \pm 2.87^{\circ}($ Fig 3$)$.

The mean coronal angular measuredinal course of the nerves, while thicker slab MIP reconstructions resulted in blurring of the nerves due to overlapping of adjacent structures. In our initial testing, 20-mm-thick slabs provided the optimal longitudinal depiction of the nerves; MIP parameters would not be expected to change substantially in patients with conditions such as chronic inflammatory demyelinating polyneuropathy, or neurofibromatosis, though parameters could be optimized for each patient.

The sagittal plane measurements for the angle ventral to the thecal sac were denoted by positive numbers and dorsal to the thecal sac, by negative numbers. Femoral and sciatic nerve calibers were measured in their midportions in the abdomen and ments for the sagittal long axis demonstration of the right femoral nerve were $33.54^{\circ} \pm 4.55^{\circ}$ and $33.87^{\circ} \pm 3.57^{\circ}$; and for the left femoral nerve, $32.85^{\circ} \pm 4.24^{\circ}$ and $34.29^{\circ} \pm 3.75^{\circ}$, respectively (Fig 4). The mean coronal angular measurements for the sagittal long axis demonstration of the right sciatic nerve were $33.01^{\circ} \pm$ $4.41^{\circ}$ and $34.53^{\circ} \pm 3.8^{\circ}$ and, for the left sciatic nerve, $32.75^{\circ} \pm 3.72^{\circ}$ and $34.37^{\circ} \pm 3.5^{\circ}$, respectively (Fig 5 ). The interobserver ICC was moderate $(0.582-0.671)$ for various angular measurements. For intraobserver performance among various angular measurements, the ICC was moderate to good (0.586-0.788).

Femoral nerve caliber on MR imaging was almost half that of 

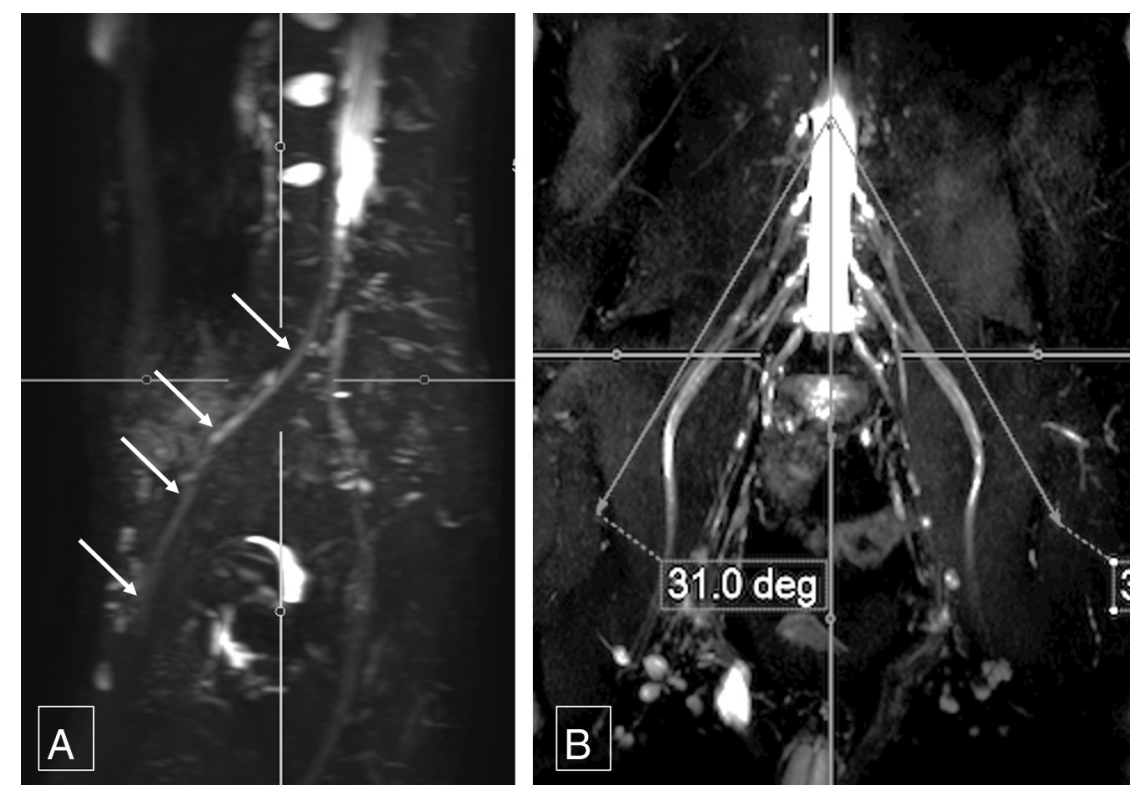

FIG 5. Coronal femoral nerve angle. Thick-slab 20-mm sagittal MIP image from a 3D SHINKEI acquisition $(A)$ obtained at a coronal angle of $31^{\circ}(B)$ optimally demonstrates the right femoral nerve (arrows, $A$ ) from its origin to the maximum length. Deg indicates degree.
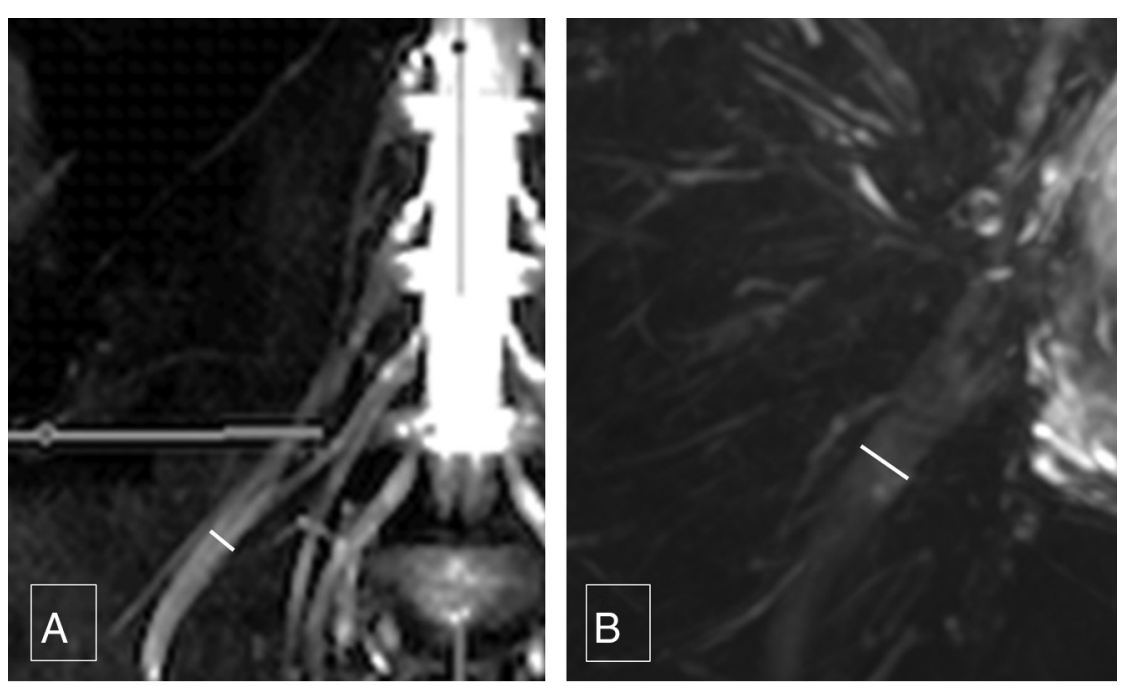

FIG 6. Femoral and sciatic nerve calibers. Thick-slab 20-mm sagittal MIP images from a 3D SHINKEl acquisition through the abdomen $(A)$ and pelvis $(B)$ show the femoral nerve diameter (4.8 $\mathrm{mm})$, which is almost one-half of sciatic nerve diameter $(9.7 \mathrm{~mm})$.

\section{ICC: intraobserver performance}

\begin{tabular}{lc}
\hline \multicolumn{1}{c}{ Variable } & Intraobserver ICC \\
\hline Fem cor Lt & 0.75146 \\
Fem cor Rt & 0.69077 \\
Fem sag & 0.78773 \\
Fem width Lt & 0.64571 \\
Fem width Rt & 0.75638 \\
L plexus sag & 0.67175 \\
Sciatic width Lt & 0.39228 \\
Sciatic width Rt & 0.73182 \\
Sciatic cor Lt & 0.58572 \\
Sciatic cor Rt & 0.61695 \\
Sciatic sag & 0.72101 \\
\hline
\end{tabular}

Note:-Fem indicates femoral; cor, coronal; Lt, left; Rt, right; L, lumbrosacral; sag, sagittal. the sciatic nerve. Mean right femoral nerve thickness was $4.52 \pm 1.11 \mathrm{~mm}$ and $4.85 \pm 0.64 \mathrm{~mm}$ for the 2 observers, and mean left femoral nerve thickness was $4.48 \pm 0.97 \mathrm{~mm}$ and $4.94 \pm 0.57 \mathrm{~mm}$, respectively. Mean right sciatic nerve thickness was $9.71 \pm 1.76 \mathrm{~mm}$ and $9.94 \pm 0.83 \mathrm{~mm}$, and mean left sciatic nerve thickness was $10.03 \pm 1.71 \mathrm{~mm}$ and $9.98 \pm 0.99 \mathrm{~mm}$, respectively (Fig 6). The interobserver ICC was moderate to good (0.557-0.737) for the above thicknesses except for the right femoral nerve thickness, which was poor (0.343). Intraobserver performance for nerve thickness was moderate to good (0.646$0.756)$, except for the left sciatic nerve thickness, which was poor (0.392) (Table).

\section{DISCUSSION}

3D SHINKEI allows excellent vascular and fat signal suppression, leading to selective nerve depiction. ${ }^{6,7}$ This sequence uses adiabatic inversion recovery for uniform fat suppression and a motionsensitive driven equilibrium pulse for vascular signal suppression, thereby enabling selective demonstration of the LS plexus nerves. ${ }^{5}$ The variable turbo spinecho component limits the acquisition time to 7-8 minutes.

This imaging technique allows ready depiction of the nerves in various arbitrary planes. Thus, the angular prescriptions could be easily and confidently measured $^{8,9}$; these results are reflected in nearly good interobserver and intraobserver performance in angular measurements.

The femoral nerve calibers were $\sim 4-5 \mathrm{~mm}$, the sciatic nerve calibers were $\sim 8-11 \mathrm{~mm}$, and the interobserver performance was moderate to good except for the right femoral nerve, due to the relatively smaller size of the femoral nerve. The normative data can be used for identification of pathologies that frequently cause abnormal nerve thickening, such as chronic demyelinating polyneuropathy, diabetic polyneuropathy, perineurioma, or Charcot-Marie-Tooth disease and so forth. ${ }^{10-12}$ The nerve-caliber measurements are also reproducible, as shown by moderate-to-good ICC results for intraobserver performance, except for the left sciatic nerve caliber, which was poor (0.392).

Although this is the first study depicting angular and nervecaliber measurements, there are some limitations. We did not obtain surgical or cadaveric correlation because it would not be practical to obtain such correlations for a large sample. In addi- 
tion, no correlations with diffusion imaging were obtained, which is also shown to produce nerve-selective images. The observers felt confident in nerve identification on 3D SHINKEI during the evaluation of the initial training set due to excellent vascular signal suppression and nerve depiction by this technique. It was therefore decided that diffusion imaging evaluation can be avoided and left as the subject of another article. Another limitation is that we evaluated only patients with normal nerves. Patients with significant nerve pathology/known mass lesions were excluded from our patient population. Patients with significant nerve pathology may require different angles to optimize the longitudinal display of the nerves, due to distortion of nerve course. Nerve caliber in these patients would also be expected to differ from that of patients with normal nerves. Finally, we did not measure obturator nerve angles. Because this nerve has been shown to travel in a straight coronal plane, it was thought that further study would not yield a significant angle. ${ }^{13}$

\section{CONCLUSIONS}

The study fills a gap in the literature regarding angular LS plexus nerve measurements for their maximum length depiction and nerve thickness. It will aid in prescription of predictable nerve planes on MR imaging for their optimal longitudinal demonstration.

\section{ACKNOWLEDGMENTS}

The authors would like to acknowledge Dr Vibhor Wadhwa for help with image formatting.

Disclosures: Avneesh Chhabra—UNRELATED: Consultancy: Siemens (CAD Consulting); Royalties: Jaypee, Wolters Kluwer, Elsevier, Comments: book royalties; Payment for Development of Educational Presentations: educational symposia; OTHER: A. Chhabra has received research grants from GE-Radiology Research Academic Fellowship, Siemens, Gatewood Fellowship Award, and Integra Life Sciences.

\section{REFERENCES}

1. Neufeld EA, Shen PY, Nidecker AE, et al. MR imaging of the lumbosacral plexus: a review of techniques and pathologies. J Neuroimaging 2015;25:691-703 CrossRef Medline
2. Soldatos T, Andreisek G, Thawait GK, et al. High-resolution 3-T MR neurography of the lumbosacral plexus. Radiographics 2013;33: 967-87 CrossRef Medline

3. Delaney H, Bencardino J, Rosenberg ZS. Magnetic resonance neurography of the pelvis and lumbosacral plexus. Neuroimaging Clin $\mathrm{N}$ Am 2014;24:127-50 CrossRef Medline

4. Vargas MI, Gariani J, Delattre BA, et al. Three-dimensional MR imaging of the brachial plexus. Semin Musculoskelet Radiol 2015;19: 137-48 CrossRef Medline

5. Kasper JM, Wadhwa V, Scott KM, et al. SHINKEI-a novel 3D isotropic MR neurography technique: technical advantages over 3DIRTSE-based imaging. Eur Radiol 2015;25:1672-77 CrossRef Medline

6. Yoneyama M, Takahara T, Kwee TC, et al. Rapid high resolution MR neurography with a diffusion-weighted pre-pulse. Magn Reson Med Sci 2013;12:111-19 CrossRef Medline

7. Huisman M, Staruch RM, Ladouceur-Wodzak M, et al. Non-invasive targeted peripheral nerve ablation using 3D MR neurography and MRI-guided high-intensity focused ultrasound (MR-HIFU): pilot study in a swine model. PLoS One 2015:14;10:e0144742 CrossRef Medline

8. Burge AJ, Gold SL, Kuong S, et al. High-resolution magnetic resonance imaging of the lower extremity nerves. Neuroimaging Clin $\mathrm{N}$ Am 2014;24:151-70 CrossRef Medline

9. Chhabra A, Rozen S, Scott K. Three-dimensional MR neurography of the lumbosacral plexus. Semin Musculoskelet Radiol 2015;19: 149-59 CrossRef Medline

10. Ellegala DB, Monteith SJ, Haynor D, et al. Characterization of genetically defined types of Charcot-Marie-Tooth neuropathies by using magnetic resonance neurography. J Neurosurg 2005;102:242-45 CrossRef Medline

11. Mauermann ML, Amrami KK, Kuntz NL, et al. Longitudinal study of intraneural perineurioma: a benign, focal hypertrophic neuropathy of youth. Brain 2009;132(pt 8):2265-76 CrossRef Medline

12. Lozeron $P$, Lacour MC, Vandendries C, et al. Contribution of plexus MRI in the diagnosis of atypical chronic inflammatory demyelinating polyneuropathies. J Neurol Sci 2016;360:170-75 CrossRef Medline

13. Petchprapa CN, Rosenberg ZS, Sconfienza LM, et al. MR imaging of entrapment neuropathies of the lower extremity, Part 1: the pelvis and hip. Radiographics 2010;30:983-1000 CrossRef Medline 